\title{
Efeitos de área e de borda sobre a estrutura florestal em fragmentos de floresta de terra-firme após 13-17 anos de isolamento
}

\author{
Henrique E. M. NASCIMENTO1, William F. LAURANCE ${ }^{1,2}$
}

\section{RESUMO}

As estimativas de densidade e biomassa de árvores vivas com DAP $\geq 10 \mathrm{~cm}$ e arvoretas 1-9,9 $\mathrm{cm}$ de DAP, liteira lenhosa grossa caída (LCG - diâmetro $\geq 10 \mathrm{~cm}$ ), árvores mortas em pé ( $\geq 10$ de DAP) e liteira lenhosa fina caída (LCF - 2,5 - 9,9 cm de diâmetro) foram quantificadas em 56 parcelas permanentes de 1 ha, distribuídas em quatro categorias de tamanho de fragmento - fragmentos de 1 ha (4 parcelas), fragmentos de 10 ha (12 parcelas) e fragmentos de 100 ha (14 parcelas) e floresta contínua (19 parcelas) e em duas classes de distância da borda - <300 m de distância da borda (29 parcelas) e > $300 \mathrm{~m}$ ( 21 parcelas). A densidade e a biomassa de árvores e arvoretas de espécies de estágios sucessionais mais avançados não diferiram significativamente entre as diferentes categorias de tamanho e entre as duas distâncias da borda. Por outro lado, fragmentos florestais e locais $<300 \mathrm{~m}$ de distâcia da borda tiveram maior biomassa e densidade de árvores e arvoretas de espécies pioneiras do que floresta contínua e locais $>300 \mathrm{~m}$ da borda, respectivamente. Fragmentos florestais apresentaram maior quantidade de LCG e LCF do que a floresta contínua. Houve também diferenças significativas entre ambas as distâncias da borda para a quantidade de LCG e LCF e necromassa total. Uma análise de covariância mostrou que não houve efeito de tamanho do fragmento, mas a distância da borda teve um efeito significativo sobre a quantidade de LCG e LCF. A quantidade de LCG e LCF foi correlacionada negativamente com a distância da borda - locais mais próximos à borda tiveram cerca de $40 \%$ e $60 \%$ mais LCG do que locais mais distantes.

\section{PALAVRAS-CHAVE}

Amazônia, Fragmentação Florestal, Efeitos de Borda, Biomassa Vegetal.

\section{Area and edge effects on forest structure in Amazonian forest fragments after 13-17 years of isolation}

\begin{abstract}
Density and biomass of live trees $\geq 10 \mathrm{~cm} \mathrm{DBH}$ and saplings $1-9.9 \mathrm{~cm} \mathrm{DBH}$, coarse woody debris (LCG-diameter $\geq 10 \mathrm{~cm}$ ), fine woody debris ( $L C F$-diameter 2.5-9.9 cm), and standing dead trees $(\geq 10 \mathrm{~cm} \mathrm{DBH)}$ were quantified in 56 permanent, 1-ha sample plots. These plots are located in four 1- (4 plots), three 10-(12 plots) and two 100-(14 plots) forest fragments in size and nearby continuous forests (19 plots) as well as in two classes of distance from the edges - $<300 \mathrm{~m}$ (29 plots) and $>300 \mathrm{~m}$ (21 plots). Density and biomass of primary species did not differ significantly among the four size categories and the two edge distance classes. However, forest fragments and distance $<300 \mathrm{~m}$ from the edges had more biomass and density of pioneer trees and saplings than did continuous forest and distance $>300 \mathrm{~m}$ from the edge, respectively. There were no significant differences among the size categories for standing dead trees. Forest fragments, however, had more quantity of LCG and LCF than did continuous forests. Moreover, distances $<300 \mathrm{~m}$ from the edges had higher quantity of LCG and LCF and total necromass than did distances $>300 \mathrm{~m}$. We performed an ANCOVA to assess whether differences in LCG and LCF in fragments were due to proximity of forest borders. An ANCOVA showed that there was no significant effect of fragment size on necromass, but a significant effect of edge distance on both LCG and LCF. The quantity of LCG and LCF was correlated negatively with edge distance - sites close to the edge presented over 40-60\% more LCG than sites far from the edges in both forest fragments and continuous forests.
\end{abstract}

\section{KEY WORDS}

Amazonia, Forest Fragmentation, Edge Effects, Area Effects, Forest Biomass.

\footnotetext{
${ }_{1}^{1}$ Projeto Dinâmica Biológica de Fragmentos Florestais, INPA/PDBFF, CP 478, 69.011-970, Manaus-AM, Brasil. Fax: (92) 3642 2050, tel: 3642 1148, e-mail:henrique@inpa.gov.br ${ }^{2}$ Smithsonian Tropical Research Institute, Apartado 2072, Balboa, República de Panamá
} 


\section{INTRODUÇÃO}

A fragmentação florestal causa muitas mudanças físicas e ecológicas como resultado da perda e isolamento de habitat (Lovejoy et al., 1986; Bierregaard et al., 1992). Conforme as paisagens florestais tornam-se fragmentadadas, as populaçōes das espécies são reduzidas, os padrōes de migração e dispersão são alterados e os habitats tornam-se expostos à condiçōes externas adversas anteriormente inexistentes, o que resulta, em última análise, numa deterioração da diversidade biológica ao longo do tempo (Tilman et al., 1994; Terborgh et al., 1997). Os efeitos de borda e os efeitos de área são os mais importantes fatores que levam às mudanças em comunidades fragmentadas. Os efeitos de área referem-se às mudanças ecológicas que ocorrem em função do isolamento do fragmento e são proporcionais à area do fragmento. Por exemplo, as espécies que ocorrem naturalmente em baixa densidade podem sofrer considerável redução do tamanho populacional em fragmentos pequenos e além disso tornam-se mais vulnerávies à extinção local devido a eventos estocásticos demográficos, catastróficos e genéticos (Shafer, 1981). Os efeitos de borda, por outro lado, são causados por gradientes diferenciados de mudanças fisícas e bióticas próximos às bordas florestais e, portanto, são proporcionais à distância da borda mais próxima (Lovejoy et al., 1986; Murcia, 1995). Ambos operam paralelamente, já que quanto menor o tamanho de um fragmento florestal maior é a razão borda/área e portanto fragmentos menores estão mais sujeitos a maiores intensidades dos efeitos de borda (Zuidema et al., 1996).

Os efeitos de borda vêm sendo relativamente bem avaliados em fragmentos de floresta em regiōes tropicais no tocante às comunidades de plantas. A criação de bordas florestais expostas a locais abertos leva à modificaçôes nas condiçôes microclimáticas (Kapos, 1989; Williams-Linera, 1990; Camargo \& Kapos, 1995) e aumento da turbulência de ventos (Laurance, 1997), resultando num aumento nas taxas de mortalidade e danos de árvores e conseqüente abertura de clareiras próximos às bordas (Williams-Linera, 1990; Ferreira \& Laurance, 1997; Laurance et al., 1998a). Conseqüentemente, mudanças na abundância relativa e composição de espécies de plantas podem ocorrer, em grande parte devido ao aumento no recrutamento e densidade de espécies arbóreas pioneiras (Williams-Linera, 1990; Laurance et al. 1998b; Sizer \& Tanner, 1999), aumento na densidade de cipós adaptadas a locais degradados (Laurance et al., 2001) e diminuição na densidade de plântulas de espécies tardias (Benitez-Malvido, 1998).

Este estudo avaliou as mudanças ocorridas na estrutura florestal no tocante ao tamanho de fragmento e distância da borda após 13-17 anos de isolamento de fragmentos de floresta de terra-firme na Amazônia central. O estudo foi organizado a fim de responder às seguintes questões: i) há diferenças entre as diferentes categorias de tamanho de fragmento com respeito às mudanças ocorridas na estrutura florestal após 13-17 anos de isolamento dos fragmentos de floresta? ii) qual é o efeito da criação de bordas sobre a estrutura florestal? e iii) em paisagens recémfragmentadas os efeitos de borda representam um papel determinante em mundanças da estrutura florestal?

\section{MÉTODOS}

\section{ÁREA DE ESTUDO E DELINEAMENTO EXPERIMENTAL}

A área de estudo, denominada de Projeto Dinâmica Biológica de Fragmentos Florestais (PDBFF), é uma paisagem experimental fragmentada administrada pelo Smithsonian Institution (SI) e Instituto Nacional de Pesquisas da Amazônia (INPA). Ela está localizada na Amazônia central brasileira cerca de $80 \mathrm{~km}$ ao norte de Manaus, $\mathrm{AM}\left(2^{\circ} 30^{\prime} \mathrm{S}, 60^{\circ} \mathrm{O}\right)$. A vegetação local é denominada como floresta de terra-firme (não sazonalmente inundada) e está a uma elevação média de 100-150 m acima do nível do mar. A precipitação média anual é de $2.200 \mathrm{~mm}$ com uma pronunciada estação seca de julho a outubro. O dossel da floresta está cerca de 30-37 m de altura, com algumas árvores emergentes podendo alcançar 55 metros. A riqueza de espécies arbóreas $(\geq 10 \mathrm{~cm}$ de DAP) pode exceder 280 espécies por hectare (Oliveira \& Mori, 1999). Os solos na área de estudo são altamente intemperizados, argilosos, ácidos e muito pobres em nutrientes como P, Ca e Ke com relativamente baixa capacidade de retenção de água (Laurance et al., 1999).

O PDBFF cobre uma área de aproximadamente $20 \mathrm{~km}$ no sentido norte-sul por $50 \mathrm{~km}$ no sentido leste-oeste e está circundada por grandes extensões de floresta contínua nos lados norte, leste e oeste. No início e meados dos anos 80 , fragmentos de floresta de 1,10 e 100 ha de tamanho e de formato quadrangular foram isolados dentro de três fazendas de gado a partir da derrubada e queima da floresta circundante e encontram-se localizados entre 70 a $650 \mathrm{~m}$ de distância da floresta contínua mais próxima (Bierregaard et al., 1992; Lovejoy et al., 1986). Devido à diminuição na produtividade dos pastos ao longo dos anos, os proprietários foram gradualmente abandonando a atividade pecuária e atualmente áreas de florestas secundárias dominadas pelos genêros Cecropia e Vismia dominam a matriz que circundam os fragmentos (Mesquita et al., 1999).

Desde o início e meados dos anos 80 (entre julho de 1980 e abril de 1985), um estudo de longo prazo sobre a mortalidade, danos, crescimento, recrutamento e composição arbórea vem sendo conduzido em florestas fragmentadas e contínuas na área de estudo. Cerca de 62.000 árvores com DAP $\geq 10 \mathrm{~cm}$ vem sendo monitoradas em intervalos regulares de 4 a 6 anos em 66 parcelas quadradas (100 X $100 \mathrm{~m}$ ) de 1 ha (Rankin de Merona et al., 1992; Laurance et al., 1998a). O último inventário nas parcelas foi realizado entre outubro de 1997 e junho de 1999. Para este estudo, selecionamos aleatoriamente 50 das 66 parcelas permanentes de 1 ha de tamanho utilizando dados deste último levantamento, que resulta num período de 13-17 anos de isolamento dos fragmentos. Deste total, 30 parcelas estão 
localizadas em nove fragmentos de floresta (quatro parcelas em quatro fragmentos de 1 ha de tamanho, 12 parcelas em três fragmentos de 10 ha e 14 em dois fragmentos de $100 \mathrm{ha}$ ) e as 20 parcelas restantes localizadas em área de floresta contínua ao longo das três fazendas. Em fragmentos florestais de 10 e 100 ha as parcelas permanentes estão estratificadas de modo que borda $\mathrm{e}$ interior estão ambos amostrados. A distância à borda mais próxima foi medida a partir do centro de cada parcela.

Devido ao fato de que no desenho experimental original a floresta contínua apresenta apenas uma parcela localizada próximo à borda $(<100 \mathrm{~m})$, neste estudo alocamos seis parcelas adicionais em áreas de borda de floresta contínua de modo que as comparaçōes dos efeitos de borda não se limitassem apenas às bordas de framentos de 10 e 100 ha. Estas seis parcelas foram alocadas em duas diferentes fazendas (quatro parcelas na fazenda Dimona e duas parcelas na fazenda Esteio) a fim de que se obtivesse uma ampla cobertura espacial da área de estudo.

\section{OBTENÇÃO DOS DADOS}

\section{Árvores $\geq 10 \mathrm{~cm}$ de DAP e Arvoretas 1-9,9 cm de DAP}

Nas 50 parcelas permanentes, os indivíduos (incluindo palmeiras) que tiveram seus DAP's (diâmetro medido à altura de $1,3 \mathrm{~m}$ acima do solo) $\geq 10 \mathrm{~cm}$ foram medidos entre os anos de 1997 a 1999. A seqüência dos levantamentos foi feita de forma que cada fragmento fosse inventariado por completo, passando ao próximo fragmento quando todas as parcelas (borda e interior) tivessem sido medidas. Para fins de análises de efeitos de tamanho e efeitos de borda (veja abaixo) este componente foi posteriormente subdividido em dois grupos: árvores de espécies de estágio sucessionais avançados e árvores de espécies pioneiras comuns em florestas secundárias (capoeiras) na área de estudo pertencentes aos gêneros Cecropia, Pourouma (Cecropiaceae), Vismia (Clusiaceae), Miconia (Melastomataceae), Laetia (Falcourtiaceae) e Goupia (Celastraceae). Os diâmetros das arvoretas $(\geq 1 \mathrm{~cm}$ de DAP e $<10 \mathrm{~cm}$ de DAP) foram medidos em 13 subparcelas de $20 \mathrm{X} 20 \mathrm{~m}$ dentro de cada parcela entre os meses de abril a outubro de 2000. Este componente foi também classificado nos dois grupos de espécies descritos acima.

Os diâmetros medidos foram convertidos às estimativas de biomassa seca acima do solo usando o modelo alométrico desenvolvido por Chambers et al. (2001) para espécies tardias ( $\geq 5 \mathrm{~cm}$ de DAP), Nascimento $\&$ Laurance (2002) para espécies tardias com DAP $\geq 1 \mathrm{~cm}$ e DAP $<5 \mathrm{~cm}$ e Nelson et al. (1999) para espécies pioneiras.

\section{NECROMASSA}

Neste estudo, a necromassa foi dividida em três componentes: i) a liteira lenhosa caída grossa (LCG) que compreende todo o material lenhoso em processo de decomposição caído sobre o solo com diâmetro ${ }^{3} 10 \mathrm{~cm}$, ii) as árvores mortas em pé com DAP $310 \mathrm{~cm}$ e iii) a liteira lenhosa caída fina (LCF) que compreende o material lenhoso também caído sobre o solo com diâmetro entre 2,5 a 9,9 cm. As estimativas da LCG e LCF para as 50 parcelas permanentes e para as seis parcelas adicionais alocadas em bordas de floresta contínua foram feitas através do método do plano de intersecção (Van Wagner, 1968; Brown, 1974) entre os meses de agosto de 1999 a abril de 2000. Dentro de cada quadrado de $20 \times 20 \mathrm{~m}$, foram estabelecidos dois planos de intersecção de $15 \mathrm{~m}$ de comprimento perpendiculares entre si (total de 26 por parcela). As partículas com diâmetro entre 2,5 a $9,9 \mathrm{~cm}$ (LCF-liteira lenhosa fina caída) foram amostradas nos primeiros cinco metros de cada plano, enquanto que as partículas com diâmetro ${ }^{3} 10 \mathrm{~cm}$ (LCG-liteira lenhosa grossa caída) foram amostradas em todo o comprimento do plano. Todas as partículas tiveram seus diâmetros medidos no ponto em que elas interseptavam o plano. A LCG foi inicialmente separada no campo em material em estágio inicial de decomposição e material em estágio avançado de decomposição, usando como critério o estado de decomposição do alburno e do cerne (Cummings 1998; Delaney et al., 1998; Graça et al., 1999). A biomassa seca para LCG e LCF foi estimada separadamente através das fórmulas fornecidas por Brown (1974), usando como densidade da madeira o valor de $0,69 \mathrm{~g} \mathrm{~cm}^{-3}$ para LCG em estágio inicial de decomposição (densidade média da madeira para a floresta Amazônica; Fearnside, 1997), 0,34 $\mathrm{g} \mathrm{cm}^{-3}$ para LCG em estágio avançado de decomposição (Cummings, 1998) e $0,41 \mathrm{~g} \mathrm{~cm}^{-3}$ para LCF (Cummings, 1998).

As estimativas de biomassa de árvores mortas em pé para as 56 parcelas foram feitas usando o modelo de Chambers et al. (2001), com uma redução de $10 \%$ dos valores para compensar as perdas de folhas e galhos (Delaney et al. 1998). Para as árvores mortas que tiveram seus troncos quebrados, uma função de forma foi usada a fim de estimar o volume (Volume = basal area X altura estimada X 0,78 (fator de correção); Graça et al., 1999), o qual foi multiplicado pela densidade média da madeira $\left(0,69 \mathrm{~g} \mathrm{~cm}^{-3}\right)$ para se obter a estimativa da biomassa.

\section{ANÁLISES ESTATÍSTICAS}

O impacto da fragmentação florestal sobre os diferentes componentes da estrutura florestal descritos acima foi avaliado através das seguintes comparações:

1) As comparações das variáveis respostas entre as diferentes categorias de tamanho de fragmento (fragmento de 1 ha, fragmento de 10 ha, fragmento de 100 ha e floresta contínua) foram feitas pelas médias das variáveis em cada categoria de tamanho através da análise de variância com um fator (ANOVA "one-way") seguido pelos testes de Tukey. Para efeito de comparaçōes entre as diferentes categorias de tamanho, as parcelas de floresta contínua nesta análise estavam localizadas com distância $>300 \mathrm{~m}$ da borda.

2) Os efeitos de borda foram avaliados a partir da subdivisão em duas categorias discretas de distância da borda $(<300 \mathrm{~m}$ e $>$ $300 \mathrm{~m}$ ) da borda, comparando as médias das variáveis respostas 
nestas duas categorias através do teste $t$ de student.

3) A análise de covariância (ANCOVA) foi usada a fim de testar se os efeitos de borda são as causas principais de mudanças ocorridas na estrutura florestal. Para tal, utilizamos as estimativas de necromassa dos diferentes componentes (LCG, árvores mortas em pé e LCF) que foram obtidas nas parcelas permanentes de fragmentos florestais e floresta contínua e nas sete parcelas adicionais alocadas em bordas de floresta contínua. Neste caso, A ANCOVA teve o efeito do tamanho (fragmento florestal e floresta contínua) como o fator fixo e distância da borda como a covariável. A distância da borda para esta análise foi subdividida em parcelas localizadas $<100 \mathrm{~m}$ e parcelas localizadas $>100 \mathrm{~m}$ da borda. Devido ao fato de que fragmentos de 10 ha e de 100 ha não se diferenciaram para quaisquer variáveis analisadas anteriormente, ambas as categorias de fragmentos foram consideradas conjuntamente para esta análise. Isto resultou num aumento no número de repetições.

Antes das análises, transformações logarítmicas $\left(\log _{10}\right)$ para aumentar a probabilidade de uma distribuição normal e reduzir a heterocedasticidade de variâncias foram feitas naquelas variáveis que exibiram distribuiçôes assimétricas. Todas as análises estatísticas foram realizadas no SAS versão 8.01 (SAS Institute, 1997).

\section{RESULTADOS}

\section{DENSIDADE EM FUNÇÃO DO TAMANHO DE FRAGMENTO E DISTÂNCIA DA BORDA}

A densidade de árvores grandes de espécies tardias não se diferenciou entre as categorias de tamanho de fragmento nas três classes de DAP (Tabela 1). No entanto, fragmentos de 1 ha tiveram maior densidade de árvores de espécies pioneiras na classe 10-20 cm de DAP do que em fragmentos de 10 e $100 \mathrm{ha}$, e estes tiveram maior densidade do que na floresta contínua. Nesta classe de DAP, a densidade de espécies pioneiras variou de 10,9 indivíduos $\mathrm{ha}^{-1}$ na floresta contínua à 58,5 indivíduos $\mathrm{ha}^{-1} \mathrm{em}$ fragmentos de 1 ha. Não houve diferenças para as classes 20-40 e > $40 \mathrm{~cm}$ de DAP, embora fragmentos de 1 ha apresentasse uma maior densidade de espécies pioneiras em ambas as classes. Como

Tabela 1 - Comparação da estrutura florestal entre fragmentos 1 ha, 10 ha e 100 ha e floresta contínua na Amazônia central. 0s valores mostrados representam as médias e seus respectivos desvios padrões. As diferentes letras sobrescritas na mesma linha denotam diferenças significativas entre as classes de tamanho de fragmento no nível de $P<0,05$ utilizando 0 teste de Tukey.

\begin{tabular}{|c|c|c|c|c|}
\hline & Fragmento 1 ha $(n=4)$ & Fragmento 10 ha $(n=12)$ & Fragmento 100 ha $(n=14)$ & Flor. Contínua $(n=19)$ \\
\hline \multicolumn{5}{|l|}{ Árvores $\geqslant 10 \mathrm{~cm}$ DAP (No. ind. ha-1) } \\
\hline \multicolumn{5}{|l|}{$10-20 \mathrm{~cm} \mathrm{DAP}$} \\
\hline Espécies Tardias & $422,3(86,0)^{\mathrm{a}}$ & $58,5(35,6)^{\mathrm{a}}$ & $376,7(83,0)^{\mathrm{a}}$ & $30,5(39,1)^{b}$ \\
\hline Espécies Pioneiras & $422,4(28,7)^{\mathrm{a}}$ & $26,2(26,1)^{\mathrm{b}}$ & $370,1(26,5)^{\mathrm{a}}$ & $10,9(10,7)^{\mathrm{c}}$ \\
\hline \multicolumn{5}{|l|}{$20-40 \mathrm{~cm} \mathrm{DAP}$} \\
\hline Espécies Tardias & $169,5(18,0)^{\mathrm{a}}$ & $9,8(7,9)^{\mathrm{a}}$ & $166,1(19,5)^{\mathrm{a}}$ & $5,8(4,1)^{\mathrm{a}}$ \\
\hline Espécies Pioneiras & $182,9(23,9)^{\mathrm{a}}$ & $7,1(8,6)^{\mathrm{a}}$ & $165,0(21,2)^{\mathrm{a}}$ & $3,6(4,1)^{\mathrm{a}}$ \\
\hline \multicolumn{5}{|l|}{$>40 \mathrm{~cm} \mathrm{DAP}$} \\
\hline Espécies Tardias & $44,3(8,6)^{\mathrm{a}}$ & $0,8(0,9)^{\mathrm{a}}$ & $47,2(10,4)^{\mathrm{a}}$ & $0,8(1,0)^{\mathrm{a}}$ \\
\hline Espécies Pioneiras & $46,9(8,5)^{\mathrm{a}}$ & $0,5(0,9)^{\mathrm{a}}$ & $47,9(8,3)^{\mathrm{a}}$ & $0,4(0,6)^{\mathrm{a}}$ \\
\hline \multicolumn{5}{|l|}{$\overline{\text { Total }}$} \\
\hline Espécies Tardias & $636,0(65,5)^{\mathrm{a}}$ & $69,0(42,6)^{\mathrm{a}}$ & $589,9(86,8)^{\mathrm{a}}$ & $37,1(39,4)^{b}$ \\
\hline Espécies Pioneiras & $652,1(32,7)^{\mathrm{a}}$ & $35,2(32,2)^{b}$ & $583,0(43,5)^{\mathrm{a}}$ & $14,9(12,7)^{\mathrm{c}}$ \\
\hline \multicolumn{5}{|c|}{ Arvoretas 1 - 9,9 cm DAP (№. ind. ha-1) } \\
\hline Espécies Tardias & $6318,1(1407,9)^{\mathrm{a}}$ & $5063,8(1049,8)^{b}$ & $6736,3(1864,2)^{a}$ & $5135,4(884,8)^{\mathrm{b}}$ \\
\hline Espécies Pioneiras & $230,0(91,3)^{\mathrm{a}}$ & $165,9(68,0)^{\mathrm{b}}$ & $167,5(55,1)^{b}$ & $137,4(121,4)^{\mathrm{b}}$ \\
\hline \multicolumn{5}{|l|}{ Árvores $10 \mathrm{~cm} \mathrm{DAP}$} \\
\hline Espécies Tardias & $311,9(35,4)^{\mathrm{a}}$ & $323,1(31,0)^{\mathrm{a}}$ & $327,6(43,1)^{\mathrm{a}}$ & $312,4(39,2)^{\mathrm{a}}$ \\
\hline Espécies Pioneiras & $7,2(3,9)^{\mathrm{a}}$ & $4,8(2,5)^{\mathrm{ab}}$ & $4,4(3,1)^{\mathrm{ab}}$ & $2,4(1,6)^{b}$ \\
\hline \multicolumn{5}{|l|}{ Arvoretas 1 - 9,9 cm DAP } \\
\hline Espécies Tardias & $22,9(4,1)^{\mathrm{a}}$ & $18,8(2,4)^{\mathrm{a}}$ & $20,8(2,9)^{\mathrm{a}}$ & $19,3(2,2)^{\mathrm{a}}$ \\
\hline Espécies Pioneiras & $3,0(1,6)^{\mathrm{a}}$ & $1,7(1,3)^{b}$ & $1,8(1,0)^{b}$ & $1,4(0,8)^{b}$ \\
\hline \multicolumn{5}{|c|}{ Liteira lenhosa caída (> 2,5 cm diâmetro) } \\
\hline 2,5 - 9,9 cm de diâmetro & $3,8(1,0)^{\mathrm{a}}$ & $4,2(0,9)^{\mathrm{a}}$ & $3,6(1,1)^{\mathrm{a}}$ & $2,9(1,0)^{b}$ \\
\hline$\geqslant 10 \mathrm{~cm}$ de diâmetro & $34,3(22,5)^{\mathrm{a}}$ & $32,6(12,8)^{\mathrm{a}}$ & $32,7(9,2)^{\mathrm{a}}$ & $25,2(9,1)^{\mathrm{b}}$ \\
\hline Árvores mortas em pé $\geqslant 10 \mathrm{~cm}$ & $4,1(1,7)^{\mathrm{a}}$ & $6,5(3,9)^{\mathrm{a}}$ & $6,7(4,7)^{\mathrm{a}}$ & $5,1(3,3)^{\mathrm{a}}$ \\
\hline Biomassa total morta & $41,2(23,4)^{\mathrm{a}}$ & $43,4(11,7)^{\mathrm{a}}$ & $43,1(10,5)^{\mathrm{a}}$ & $35,6(9,3)^{b}$ \\
\hline
\end{tabular}


resultado da alta densidade de árvores de espécies pioneiras na classe de 10-20 cm de DAP, a densidade total de espécies pioneiras $\geq 10 \mathrm{~cm}$ de DAP foi maior em fragmentos florestais do que na floresta contínua. A densidade de arvoretas de espécies tardias (1-10 cm de DAP) foi maior em fragmentos de 1 e 100 ha do que em fragmentos de 10 ha e em floresta contínua, enquanto que fragmentos de 1 ha tiveram maior densidade de arvoretas de espécies pioneiras do que as outras categorias de tamanho (Tabela 1).

Não houve diferenças na densidade de árvores de espécies tardias entre as duas categorias de distância de borda $(<300 \mathrm{e}>$ $300 \mathrm{~m}$ ) para as três classes de DAP e densidade total (Tabela 2). A densidade de espécies pioneiras, por outro lado, foi cerca de 3 vezes maior em locais com distância $<300 \mathrm{~m}$ da borda do que mais ao interior da floresta para a classe de $10-20 \mathrm{~cm}$ de DAP. Não houve diferenças na densidade de espécies pioneiras entre ambas as distâncias da borda para a classe de $20-40 \mathrm{~cm}$ e $>40 \mathrm{~cm}$ de DAP, embora a densidade total de espécies pioneiras tenha sido maior em locais $<300 \mathrm{~m}$ da borda. Para as arvoretas de espécies tardias não houve diferença significativa entre as distâncias, mas para as arvoretas de espécies pioneiras foi observado uma maior densidade em locais $<300 \mathrm{~m}$ da borda (Tabela 2).

\section{BIOMASSA EM FUNÇÃO DO TAMANHO DO FRAGMENTO E DISTÂNCIA DA BORDA}

A estimativa de biomassa de árvores de espécies tardias $\geq 10$ cm de DAP variou apenas de 311,9 $\mathrm{Mg} \mathrm{ha}^{-1}$ a $327,6 \mathrm{Mg} \mathrm{ha}^{-1}$ entre as categorias de tamanho de fragmento e floresta contínua (Tabela 1). Portanto, não houve diferenças entre as categorias de tamanho para a biomassa de espécies tardias. Entretanto, a biomassa de árvores de espécies pioneiras foi maior em fragmento de 1 ha do que em floresta contínua. Também não houve diferenças entre as categorias de tamanho de fragmento para a biomasa de arvoretas de espécies pimárias. No entanto, fragmentos de 1 ha tiveram uma maior biomassa de espécies pioneiras do que as outras categorias de tamanho de fragmento. Fragmentos florestais tiveram maior quantidade de LCG, LCF e biomassa total morta do que a floresta contínua, ao passo que a biomassa de árvores mortas em pé não diferiu entre as categorias de tamanho (Tabela 1).

A biomassa de árvores e arvoretas de espécies tardias também não diferiu entre as distâncias da borda, mas a biomassa de espécies pioneiras diferiu para ambas as classes de DAP (Tabela 2). Além disso, locais com distância < $300 \mathrm{~m}$ da borda também tiveram significantemente maior quantidade de LCG eLCF do que locais com locais com distância $>300 \mathrm{~m}$ da borda. Conseqüentemente, a biomassa total morta também diferiu entre ambas as distâncias (Tabela 2).

A biomassa da liteira lenhosa caída grossa, árvores mortas em pé e liteira lenhosa caída fina foi também analisada para as categorias de tamanho (fragmento florestal e mata contínua) e
Tabela 2 - Comparação da estrutura florestal entre as duas classes de distância da borda em fragmentos florestais e floresta contínua na Amazônia central. Os valores mostrados representam as médias e seus respectivos desvios padrões. As diferentes letras sobrescritas na mesma linha denotam diferenças significativas entre ambas as distâncias da borda no nível de $P<0,05$ utilizando 0 teste $t$.

\begin{tabular}{|c|c|c|}
\hline & $\begin{array}{l}<300 \mathrm{~m} \text { da borda } \\
(n=29)\end{array}$ & $\begin{array}{l}>300 \mathrm{~m} \text { da borda } \\
(n=21)\end{array}$ \\
\hline \multicolumn{3}{|l|}{ Árvores $\geqslant 10 \mathrm{~cm}$ DAP (№. ind. ha-1) } \\
\hline \multicolumn{3}{|l|}{$10-20 \mathrm{~cm} \mathrm{DAP}$} \\
\hline Espécies Tardias & $396,9(68,5)^{\mathrm{a}}$ & $383,6(29,1)^{\text {a }}$ \\
\hline Espécies Pioneiras & $33,4(33,7)^{\mathrm{a}}$ & $13,0(10,7)^{\mathrm{b}}$ \\
\hline \multicolumn{3}{|l|}{$20-40 \mathrm{~cm}$ DAP } \\
\hline Espécies Tardias & $169,0(22,5)^{\mathrm{a}}$ & $174,1(22,0)^{\mathrm{a}}$ \\
\hline Espécies Pioneiras & $7,8(7,0)^{\mathrm{a}}$ & $5,4(4,7)^{\mathrm{a}}$ \\
\hline \multicolumn{3}{|l|}{$>40 \mathrm{~cm} \mathrm{DAP}$} \\
\hline Espécies Tardias & $45,6(8,5)^{\mathrm{a}}$ & $49,1(8,7)^{\mathrm{a}}$ \\
\hline Espécies Pioneiras & $0,7(0,9)^{\mathrm{a}}$ & $0,3(0,5)^{\mathrm{a}}$ \\
\hline \multicolumn{3}{|l|}{$\overline{\text { Total }}$} \\
\hline Espécies Tardias & $611,5(72,0)^{\mathrm{a}}$ & $607,3(48,1)^{\mathrm{a}}$ \\
\hline Espécies Pioneiras & $48,9(36,9)^{\mathrm{a}}$ & $18,7(12,8)^{\mathrm{b}}$ \\
\hline \multicolumn{3}{|c|}{ Arvoretas 1 - 9,9 cm DAP (№. ind. ha-1) } \\
\hline Espécies Tardias & $5759,9(1310,5)^{\mathrm{a}}$ & $5583,2(1776,2)^{a}$ \\
\hline Espécies Pioneiras & $202,9(86,0)^{\mathrm{a}}$ & $149,2(124,3)^{b}$ \\
\hline \multicolumn{3}{|l|}{ Biomassa acima do solo (Mg ha-1) } \\
\hline \multicolumn{3}{|l|}{ Árvores $\geqslant 10 \mathrm{~cm} \mathrm{DAP}$} \\
\hline Espécies Tardias & $314,9(35,6)^{\mathrm{a}}$ & $326,6(40,7)^{\mathrm{a}}$ \\
\hline Espécies Pioneiras & $5,6(2,9)^{\mathrm{a}}$ & $2,8(1,7)^{\mathrm{b}}$ \\
\hline \multicolumn{3}{|c|}{ Árvores pequenas e arvoretas 1 - 9,9 cm DAP } \\
\hline Espécies Tardias & $20,1(3,1)^{\mathrm{a}}$ & $19,6(2,3)^{\mathrm{a}}$ \\
\hline Espécies Pioneiras & $3,0(1,2)^{\mathrm{a}}$ & $1,8(0,8)^{\mathrm{b}}$ \\
\hline \multicolumn{3}{|c|}{ Liteira lenhosa caída (> 2,5 cm diâmetro) } \\
\hline 2,5 - 9,9 cm de diâmetro & $3,9(1,1)^{\mathrm{a}}$ & $3,2(0,9)^{\mathrm{b}}$ \\
\hline$\geqslant 10 \mathrm{~cm}$ de diâmetro & $34,1(12,2)^{\mathrm{a}}$ & $25,4(8,2)^{b}$ \\
\hline Árvores mortas em pé $\geqslant 10 \mathrm{~cm}$ & $5,9(3,9)^{\mathrm{a}}$ & $6,0(3,7)^{\mathrm{a}}$ \\
\hline Biomassa total morta & $43,9(12,6)^{\mathrm{a}}$ & $34,5(8,4)^{b}$ \\
\hline
\end{tabular}

distâncias da borda (< 100 e > 100 m) através da análise de covariância. A ANCOVA não mostrou um efeito isolado de tamanho de fragmentos na quantidade de LCG $(F=0,40$, $P=0,53)$, árvores mortas em pé $(F=0,08, P=0,78)$ e $\operatorname{LCF}(F=0,43$, $P=0,51$, mas houve um efeito significativo de distância da borda sobre a quantidade de LCG $(F=11,1, P=0,001)$ e para a LCF $(F=3,85, P=0,055)$. Não houve uma interação significante entre tamanho e distância da borda $(F<0,56, P>0,45$ para os três casos) indicando que a pressuposição da ANCOVA de homogeneidade de declividade não foi violada (as interações não significantes foram retiradas do modelo final da ANCOVA).

Em fragmentos florestais as parcelas com distância $<100 \mathrm{~m}$ da borda tiveram aproximadamente $40 \%$ maior quantidade de LCG $\left(35,5 \pm 9,5 \mathrm{Mg} \mathrm{ha}^{-1}\right)$ do que parcelas com distância > 100 

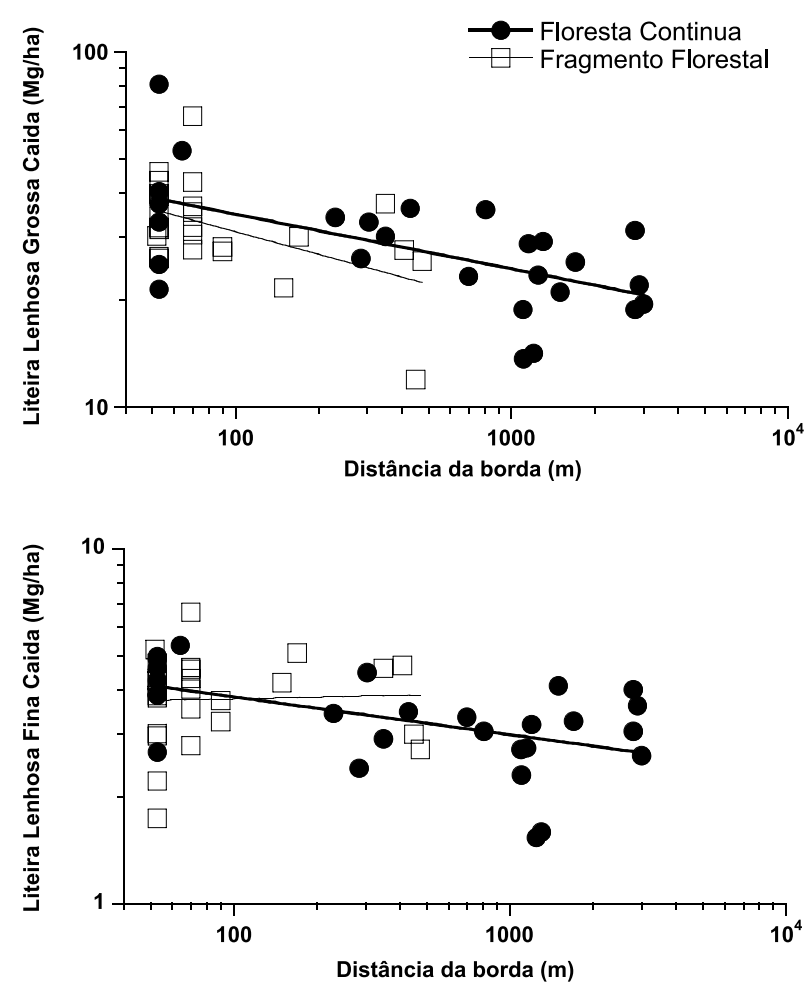

Figura 1 - Relação entre a biomassa de liteira lenhosa grossa caída e a biomassa da liteira lenhosa fina caída com a distância da borda para cada categoria de tamanho. Ambos os eixos estão em escala logaritmica.

m $\left(25,8 \pm 10,1 \mathrm{Mg} \mathrm{ha}^{-1}\right)$, enquanto que em floresta contínua esta diferença foi de cerca $60 \%\left(<100 \mathrm{~m}: 41,5 \pm 20,1 \mathrm{Mg} \mathrm{ha}^{-}\right.$ $\left.{ }^{1} \mathrm{e}>100 \mathrm{~m}: 25,5 \pm 7,1 \mathrm{Mg} \mathrm{ha}^{-1}\right)$. Não houve diferença significativa entre fragmentos florestais e floresta contínua para a distância $<100 \mathrm{~m}$ da borda $(t=0,52, P=0,48)$ e para a distância $>100 \mathrm{~m}$ da borda $(t=0,01, P=0,94)$. A maior quantidade de LCG em áreas próximas às bordas de fragmentos florestais e floresta contínua é explicada em grande parte pela diferença encontrada entre as duas distâncias da borda na classe de diâmetro $>40 \mathrm{~cm}$, na qual locais com distância $<100 \mathrm{~m}$ da borda tiveram maior quantidade de LCG do que áreas no interior (Figura 2).

\section{DISCUSSÃO}

A densidade e a biomassa de espécies tardias de árvores e arvoretas não diferiram significativamente entre as diferentes categorias de tamanho de fragmento e floresta contínua e entre as duas categorias de distância da borda. Era esperado que a densidade e biomassa de espécies arbóreas tardias com DAP $\geq 10$ $\mathrm{cm}$ diminuíssem ao longo dos anos, já que as taxas de mortalidade de árvores foram significativamente maiores em fragmentos de 1 ha e em locais com distância $<300 \mathrm{~m}$ da borda (Laurance et al. 1998a). Há duas possíveis explicações para a não diferenciação da densidade e biomassa de árvores de espécies tardias. Primeiro,

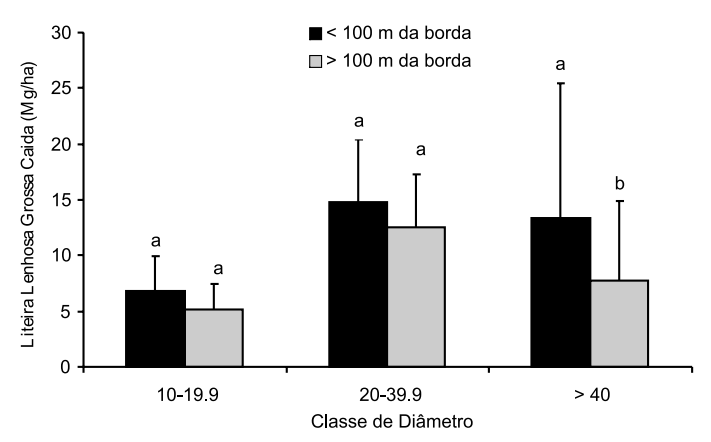

Figura 2 - Quantidade da liteira lenhosa grossa caída (média \pm desvio padrão) por classe de diâmetro para as duas classes de distância da borda ( $<100 \mathrm{~m} \mathrm{e}>100 \mathrm{~m})$ para parcelas localizadas em fragmentos florestais (10 e 100 ha) e floresta contínua. Diferentes letras indicam diferenças significativas entre as duas distâncias da borda dentro de cada classe de diâmetro $(t=5,01, P=0,02)$.

já havia anteriormente à fragmentação uma alta variação natural na biomassa de árvores com DAP $\geq 10 \mathrm{~cm}$ entre as parcelas, relacionada à fertilidade do solo e topografia (Laurance et al., 1999). Por exemplo, para 20 parcelas localizadas no interior de floresta contínua (> $300 \mathrm{~m}$ da borda), a biomassa de árvores ${ }^{3} 10$ cm de DAP variou de 305 a $432 \mathrm{Mg} \mathrm{ha}^{-1}$ (Nascimento \& Laurance, 2002). Portanto, esta variação natural tende a obscurecer os possíveis efeitos da fragmentação sobre a densidade e biomassa de árvores. Segundo, paralelamente à diminuição na densidade de árvores com o aumento nas taxas de mortalidade, vem também ocorrendo um aumento nas taxas de recrutamento de árvores de espécies secundárias em fragmentos florestais (Laurance et al., 1998b). Algumas espécies, que não foram diferenciadas neste estudo, pertencentes aos gêneros Annona, Xylopia (Annonaceae), Mabea, Croton (Euphorbiaceae), Casearia (Flacourtiaceae) e Inga (Mimosaceae) vêm aumentando suas densidades ao longo dos anos em fragmentos florestais (PDBFF, dados não publicados).

A densidade de árvores e arvoretas de espécies pioneiras muito comuns em florestas secundárias na área de estudo, por outro lado, aumentaram em fragmentos florestais e em locais próximos às bordas após 13-17 anos de fragmentação. Em florestas tropicais primárias, espécies arbóreas pioneiras são encontradas em baixa densidade, já que elas apenas colonizam grandes $\left(>200 \mathrm{~m}^{2}\right)$ clareiras (Denslow, 1987). A freqüente proliferação de clareiras em áreas próximas às bordas (Camargo \& Kapos, 1995) como resultado das altas taxas de mortalidade e danos de árvores (Ferreira \& Laurance, 1997; Laurance et al., 1998a) favorece o estabelecimento e crescimento de espécies pioneiras e secundárias adaptadas às condições de alta luminosidade. Dois estudos realizados na mesma área mostraram que os dois diferentes grupos de espécies apresentaram respostas diferenciadas com a fragmentação florestal. Sizer \& Tanner (1999) encontraram um aumento significativo nas taxas de recrutamento e crescimento de plântulas e arvoretas durante os dois primeiros anos de criação 
de uma borda, aumento este atribuído principalmente às espécies pioneiras e secundárias. Benitez-Malvido (1998), por outro lado, mostrou que a densidade de plântulas de espécies tardias declinou em locais próximos às bordas, indicando que o estabelecimento destas espécies vem diminuindo devido às condiçōes diferenciadas em áreas de borda.

Em florestas de terra-firme na Amazônia, a liteira lenhosa perfaz 6-18\% da biomassa total viva acima do solo (Delaney et al., 1998; Cummings, 1998; Uhl et al. 1988; Uhl \& Kauffman, 1990; Gerwing, 2002; Nascimento \& Laurance, 2002) e sua produção anual pode chegar até $46 \%$ da produção total de necromassa (Chambers et al., 2000). Independente do tamanho ou distância da borda, este estudo mostrou que a proporção da lenhosa lenhosa grossa compreendida pelas árvores mortas em pé foi relativamente baixa em comparação à LCG (10-15\% do total, Tabelas 1 e 2). Diferentes estudos, no entanto, mostram que a proporção dos dois componente da liteira lenhosa grossa diferencia-se entre regiōes. Na Amazônia venezuelana (Delaney et al., 1998) e na Amazônia oriental brasileira (Gerwing, 2002) a proporção de árvores mortas em pé compreendeu cerca de 40$50 \%$ da liteira lenhosa grossa, enquanto que numa área de floresta próxima (Summers, 1998) e em Rodônia (Cummings, 1998) a proporção de árvores mortas em pé abrangeu cerca de $9 \%$ e 3\%, respectivamente. As diferenças nas proporçōes de LCG e árvores mortas em pé entre locais podem ser explicadas pelas diferenças na forma predominante de mortalidade, que por sua vez pode estar relacionada às diferenças na topografia e intensidades de vento. $\mathrm{Na}$ área de estudo, a mortalidade de árvores através do vento e pela queda de outras árvores, que resulta na queda das árvores sobre o solo devido à quebra de troncos ou mesmo pela exposição da raiz, são as principais formas de mortalidade, ao passo que as árvores que morrem mas que permanencem em pé compreeendem uma baixa proporção (D'Angelo et al., 2004; Chambers et al., 2000). Num estudo comparativo entre duas florestas tropicais de terrras baixas, Borneo e Amazônia equatoriana, Gale (2000) mostrou que a proporção do estoque liteira lenhosa compreendida pelas árvores mortas em pé na Amazônia equatoriana foi muito mais baixa do que em Borneo. $\mathrm{O}$ autor atribui a isto às diferenças de declividade do terreno e de intensidades de vento entre locais.

Devido ao fato de que no interior da floresta contínua a proporção da biomassa compreendida pelas árvores mortas em pé já é relativamente baixa, e como fragmentos florestais estão mais sujeitos as maiores intensidades de vento em função da maior exposição a locais abertos, eles são mais prováveis de apresentarem uma alta proporção de LCG. Diante disso, já era esperado a não diferença da biomassa de árvores mortas em pé para tamanho de fragmentos e distância da borda. No entanto, as quantidades de LCG e LCF foram significativamente maiores em fragmentos florestais e em locais com distância $<300 \mathrm{~m}$ da borda. Como resultado da maior taxa de mortalidade e danos de árvores (Laurance et al., 1998a) e conseqüentemente uma maior produção de liteira lenhosa grossa (Nascimento \& Laurance, 2004), a quantidade de LCG foi maior em fragmentos florestais e locais próximo às bordas após 13-17 anos de fragmentação. Conforme revelado pela ANCOVA, a quantidade de LCG foi independente do tamanho, mas tanto em fragmentos florestais de 10 e 100 ha quanto em floresta contínua a quantidade foi maior em locais próximos às bordas (Figura 1). Além disso, não houve diferenças significativas entre fragmentos florestais e floresta contínua para a quantidade de LCG para ambas as distâncias da borda. Isto sugere então que em florestas recém-fragmentadas os efeitos de bordas representam os principais mecanismos que levam às mudanças da estrutura florestal. As maiores quantidades de LCG e LCF encontradas em fragmentos florestais de 10 e 100 ha (Tabela 1) estão relacionadas às maiores quantidades encontradas em locais próximos às bordas, principalmente em função da mortalidade de árvores grandes (Figura 2). Este resultado condiz com aquele encontrado por Laurance et al. (2000) que mostraram que a mortalidade de árvores foi direcionada para as maiores árvores nos fragmentos florestais $\mathrm{da}$ área de estudo. As diferenças na quantidade de LCG entre borda e interior de fragmentos florestais e floresta contínua são comparáveis a aquela encontrada $(38 \%)$ entre floresta intacta e floresta que teve $30-58 \mathrm{~m}^{3} /$ ha de madeira extraída na Amazônia oriental (Gerwing, 2002).

Espera-se que em florestas fragmentadas o aumento da necromassa e da biomassa de espécies pioneiras podem compensar a perda de biomassa de árvores grandes. Conforme mencionado acima, de fato não houve diferenças significativas para a biomassa de árvores grandes entre fragmentos florestais e floresta contínua e entre as distâncias da borda, como resultado principalmente das diferenças na distribuição espacial da biomassa entre as parcelas na área de estudo. Isto faz com que a real perda de biomassa com a fragmentação florestal seja mascarada por esta variação natural. Num estudo de longo prazo sobre a mortalidade e o recrutamento de árvores com DAP $\geq 10 \mathrm{~cm}$ e medidas de todos os outros componentes da biomassa acima do solo, Nascimento \& Laurance (2004) mostraram a perda média líquida de biomassa nos primeiros $300 \mathrm{~m}$ da borda foi de 10,9 $\mathrm{Mg} \mathrm{ha}^{-1}$ após 13-17 anos de fragmentação utilizando as mesmas parcelas deste estudo.

A liteira lenhosa em ecossistemas tropicais apresenta uma alta taxa de decomposição (Chambers et al., 2000; Harmon et al., 1995) comparativamente a outros ecossistemas e, conseqüentemente, o tempo de permanência do carbono na liteira lenhosa é relativamente curto (média de 5,9 anos, Chambers et al., 2000) quando comparado às árvores vivas, que podem viver até 1.000 anos (Chambers et al., 1998). Aliado a isto, conforme mostrado neste estudo, a densidade e biomassa de espécies pioneiras vem aumentando em fragmentos, fazendo com que haja um aumento da taxa de decomposição, já que a densidade de madeira é correlacionada positivamente com taxa de 
decomposição (Chambers et al., 2000). De fato, baseado nos dados de longo prazo de mortalidade de árvores na área de estudo e no estoque de liteira lenhosa grossa, Nascimento \& Laurance (2004) mostraram que o "turnover" da liteira lenhosa grossa (a fração da necromassa que se decompõe anualmente) foi correlacionado negativamente com a distância da borda, sugerindo que em florestas fragmentadas a decomposição da liteira lenhosa está sendo mais rápida. Portanto, conforme a biomassa armazenada em árvores de vida longa vai sendo perdida ao longo dos anos, a posterior decomposição da necromassa será o principal mecanismo que resulta na emissão de carbono para a atmosfera.

Em paisagens tropicais recentemente fragmentadas, os efeitos de borda têm um importante papel sobre a estrutura florestal, embora as escalas espaciais possam variar consideravelmente para diferentes alteraçôes relacionadas aos efeitos de borda (Lovejoy et al., 1986; Didham \& Lawton, 1999; Laurance et al., 2002). Maiores taxas de mortalidade de árvores, por exemplo, podem ser detectadas numa distância de até $300 \mathrm{~m}$ da borda (Laurance et al., 1998a, 2000). Por outro lado, o aumento no estoque e na produção de liteira fina ocorre em menores escalas $(<100 \mathrm{~m}$ da borda), possivelmente em função das mudanças microclimáticas serem mais intensas em áreas mais próximas às bordas (Carvalho \& Vasconcelos, 1999; Didham, 1998; Didham \& Lawton, 1999).

Os efeitos de área e de borda têm sido raramente discriminados em estudos de florestas fragmentadas. Para fragmentos de mesma forma, a relação área/perímetro é maior quanto maiores são os fragmentos e, portanto, quanto maior esta relação menor a intensidade dos efeitos de borda em florestas fragmentadas (Zudeima et al., 1996). Em paisagens fragmentadas como a deste estudo, onde principalmente incêndios são controlados, os efeitos de borda relacionados às mudanças microclimáticas e maior intensidade de ventos são as principais causas que levam às mudanças na composição e estrutura de fragmentos florestais. Os fragmentos menores podem ser totalmente devastados por ventos de fortes intensidades, enquanto que em fragmentos maiores podem ocorrer perturbaçóes iniciais e o subseqüente estabelecimento de vegetação secundária em locais próximos às suas bordas (Gascon et al., 2000; Laurance et al., 2002). Estas mudanças associadas às bordas podem criar, ao longo dos anos, uma zona-tampão que pode agir contra os efeitos deletérios inicias de mudanças microclimáticas (Camargo \& Kapos, 1995; Gascon et al., 2000; Mesquita et al., 1999).

Evidências sugerem, no entanto, que a alta mortalidade e danos de árvores na área de estudo não são meramente respostas momentâneas da fragmentação às mudanças iniciais abruptas das condiçôes microclimáticas e de intensidades de ventos (Laurance et al., 1998a), pois não existe uma relação entre a idade de fragmentos florestais com as taxas de mortalidade. Isto indica que mudanças contínuas na estrutura florestal ocorrerão independentes do tempo de isolamento dos fragmentos. Portanto, conforme as árvores tardias continuem reduzindo a densidade, espécies pioneiras e secundárias serão favorecidas devido às novas condiçōes criadas. Este fato é apoiado pelos estudos realizados em fragmentos florestais mais velhos do que os deste estudo. Em fragmentos localizados na Mata Atlântica brasileira (Viana et al., 1997; Tabarelli et al., 1999) e na Malásia (Turner et al., 1996), regiōes onde o processo de fragmentação florestal iniciou-se há vários anos, os fragmentos florestais nestes locais foram caracterizados como tendo alta percentagem de clareiras e alta densidade de cipós, bambus, espécies pioneiras ruderais e presença de espécies exóticas. No entanto, deve ser enfatizado que os fragmentos de floresta nestas regiōes foram e estão sendo influenciados por atividades humanas relacionadas à atividade agro-pecuária. Neste caso, fragmentos florestais são frequentemente deteriorados a partir do uso de pesticidas, queimadas e outras práticas comuns de uso da terra (Viana et al., 1997). A maioria dos fragmentos deste estudo, ao contrário, está rodeada por vegetação secundária estabelecidas desde o início os anos 90 devido o abandono da atividade pecuária resultante da baixa produtividade das pastagens e, portanto, não estão sujeitos a quaisquer efeitos deletérios de práticas de uso da terra e ainda podem ter os efeitos positivos da matriz circundante. Os fragmentos de floresta rodeados por vegetação secundária podem experimentar uma diminuição nas taxas de mortalidade de árvores (Mesquita et al., 1999).

\section{AGRADECIMENTOS}

Este trabalho contou com o apoio financeiro do Programa National Aeronautics and Space Administration (NASA)/Large Biosphere-Atmosphere Experiment in the Amazon (NASA/ LBA), Fundação A. W. Mellon, Fundação Conservation, Food, and Health, World Wildlife Fund (WWF), Fundação MacArthur, Instituto Nacional de Pesquisas da Amazônia (INPA), Smithsonian Institution (SI) e Conselho Nacional de Desenvolvimento Científico e Tecnológico (CNPq). Esta é a publicação de número 459 da série técnica do Projeto Dinâmica Biológica de Fragmentos Florestais.

\section{BIBLIOGRAFIA CITADA}

Benitez-Malvido, J. 1998. Impact of forest fragmentation on seedling abundance in a tropical rais forest. Conservation Biology, 12:380389.

Bierregaard, R. O.; Lovejoy, T. E.; Kapos, V.; Santos, A. A. dos; Hutchings, R. W. 1992. The biological dynamics of tropical rain forest fragments. Bioscience, 42:859-866.

Brown, J. K. 1974. Handbook for inventorying downed wood debris. USDA Forest Service, Ogden, Utah, 25 pp.

Carvalho, K. S.; Vasconcelos, H. L. 1999. Forest fragmentation in central Amazonia and its effects on litter-dwelling ants. Biological Conservation, 91:151-158. 
Camargo, J. L. C.; Kapos, V. 1995. Complex edge effects on soil moisture and microclimate in central Amazonian forest. Journal of Tropical Ecology, 11:205-221.

Chambers, J. Q.; Higuchi, N.; Schimel, J. 1998. Ancient trees in Amazonia. Nature, 391:135-136.

Chambers, J. Q.; Higuchi, N.; Schimel, J. P.; Ferreira, L. V.; Melack, J. M. 2000. Decomposition and carbon cycling of dead wood in tropical forests on the central Amazon. Oecologia, 122:380388.

Chambers, J. Q.; Santos, J. dos; Ribeiro, R. J.; Higuchi, N. 2001. Tree damage, allometric relationships, and above-ground net primary production in central Amazon forest. Forest Ecology and Management, 152:73-84.

Cummings, D. L. 1998. Total aboveground biomass and structure of tropical forest delineated by Projeto RADAMBRASIL in northern Rondônia, Brazil. M.Sc. thesis, Oregon State University, Corvallis, Oregon. 123 pp.

D'Angelo, S.; Andrade, A.; Laurance S. G.; Laurance, W. F.; Mesquita, R. 2004. Inferred causes of tree mortality in fragmented and intact Amazonian forests. Journal of Tropical Ecology, 11:243:246.

Delaney, M.; Brown, S.; Lugo, A. E.; Torres-Lezama, A.; Quintero, N. B. 1998. The quantity and turnover of dead wood in permanent forest plots in six life zones of Venezuela. Biotropica, 30:2-11.

Denslow, J. S. 1987. Tropical tree-fall gaps. Annual Review Ecology and Systematics, 17:430-441.

Didham, R. K. 1998. Altered leaf-litter decomposition rates in tropical forest fragments. Oecologia, 116:397-406.

Didham, R. K.; Lawton, J. H. 1999. Edge structure determines the magnitude of changes in microclimate and vegetation structure in tropical forest fragments. Biotropica, 31:17-30.

Fearnside, P. M. 1997. Wood density for estimating forest biomass in Brazilian Amazonia: Forest Ecology and Management, 90:5987.

Ferreira, L. V.; Laurance, W. F. 1997. Effects of forest fragmentation on mortality and damage of selected tree in central Amazonia. Conservation Biology, 20: 243-246.

Gale, N. 2000. The aftermath of tree death: coarse woody debris and the topography in four tropical rain forests. Canadian Journal of Forest Research, 30:1489-1493.

Gascon, C.; Williamson, G. B.; Fonseca, G. A. B. 2000. Receding edges and vanishing reserves. Science, 288:1356-1358.

Gerwing, J. J. 2002. Degradation of forests through logging and fire in the eastern Brazilian Amazon. Forest Ecology and Management, 157:131-141

Graça, P. M. L. A.; Fearnside, P. M.; Cerri, C. C. 1999. Burning of Amazonian forest in Ariquemes, Rondônia, Brazil: biomass, charcoal formation, and burning efficiency. Forest Ecology and Management, 120:179-191.

Harmon, M. E.; Whigham, D. F.; Sexton, J.; Olmsted, I. 1995. Decomposition and mass of wood detritus in the dry tropical forests of the northeastern Yucantan peninsula, Mexico. Biotropica, 27:305-316
Kapos, V. 1989. Effects of isolation on the water status of tropical patches in the Brazilian Amazon. Journal of Tropical Ecology, 5:173-185.

Laurance, W. F. 1997. Hyper-disturbed parks: edge effects and the ecology of isolated rain forest reserves in tropical Australia. In: W. F. Laurance, R. O. Bierregaard (eds.). Tropical Forest Remnants: Ecology, Management, and Conservation of Fragmented Landscape. University of Chicago Press, Chicago, p. 71-83.

Laurance, W. F.; Ferreira, L. V.; Rankin-de-Merona, J. M.; Laurance, S. G. 1998a. Rain forest fragmentation and the dynamics of Amazonian tree communities. Ecology, 69:2032-2040.

Laurance, W. F.; Ferreira, L. V.; Rankin-de-Merona, J. M.; S. G. Laurance. 1998b. Effects of forest fragmentation on recruitment patterns in Amazonian tree communities. Conservation Biology, 12:460-464.

Laurance, W. F., Fearnside, P. M.; Laurance, S. G.; Delamonica, P.; Lovejoy, T. E.; Rankin-de-Merona, J. M.; Chambers, J. Q.; Gascon, C. 1999. Relationship between soils and Amazon forest biomass: a landscape-scale study. Forest Ecology and Management, 118:127-138.

Laurance, W. F.; Delamonica, P.; Laurance, S. G.; Vasconcelos, H. L.; Lovejoy, T. E. 2000. Rainforest fragmentation kills big trees. Nature, 404:836.

Laurance, W. F; Perez-Salicrup, D.; Delamonica, P.; Fearnside, P. M.; D’Angelo, S.; Jerozolinski, A.; Pohl, L.; Lovejoy, T. E. 2001. Rain forest fragmentation and the structure of Amazonian liana communities. Ecology, 82:105-116.

Laurance, W. F.; Lovejoy, T.; Vasconcelos, H. L.; Bruna, E. M.; Didham, R. K.; Stouffer, P.; Gascon, C.; Bierregaard, R.; Laurance, S.; Sampaio, E. 2002. Ecosystem decay of Amazonian forest fragments, a 22-year investigation. Conservation Biology. 16(3): 605-618.

Lovejoy, T. E.; Bierregaard, R. O.; Rylands, A. B.; Malcolm, J. R.; Quintela, C. E.; Harper, L. H.; Brown, K. S.; Powell, A. H.; Powell, G. V. N.; Schubart, H. O. R.; Hays, M. B. 1986. Edge and other effects of isolation on Amazon forest fragments. In: Soulé, M. E. (Ed.). Conservation Biology: The Science of Scarcity and Diversity. Sinauer, Sunderland, Massachusetts, USA. p. 257285.

Mesquita, R. C. G.; Delamonica, P.; Laurance, W . F. 1999. Effects of surrounding vegetation on edge-related tree mortality in Amazonian forest fragments. Biological Conservation, 91:129134.

Murcia, C. 1995. Edge effects in fragmented forests: implications for conservation. Trends in Ecology and Evolution, 10:58-62.

Nascimento, H. E. M.; Laurance, W. F. 2002. Total aboveground biomass in central Amazonian rainforests: a landscape-scale study. Forest Ecology and Management, 168:311-321.

Nascimento, H. E. M.; Laurance, W. F. 2004. Biomass dynamics in Amazonian forest fragments. Ecological Applications, 14 (Supplement): 127-138.

Nelson, B. W.; Mesquita, R.; Pereira, J. L. G.; Souza, S. G. A.; Batista, G. T.; Couto, L. B. 1999. Allometric regressions for 


\section{ACTA

improved estimates of secondary forest biomass in the central Amazon. Forest Ecology and Management, 117:149-167.

Oliveira, A. A.; Mori, S. 1999. A central Amazonian terra firme forest. I. High tree species richness on poor soils. Biodiversity and Conservation, 8:1219-1244.

Rankin-de-Merona, J. M.; Prance, J. M.; Hutchings, R. W.; R. W. Silva, R. W.; Rodrigues, W. A.; Uehling, M. A. 1992. Preliminary results of a large-scale inventory of upland rain forest in the central Amazon. Acta Amazonica, 22:493-534.

SAS Institute. 1997. SAS/STAT, Release 8.01 Edition. Cary, NC, USA.

Shafer, M. L. 1981. Minimum population sizes for species conservation. Bioscience, 31:131-134.

Sizer, N.; Tanner, E. V. J. 1999. Response of woody plant seedlings to edge formation in a lowland tropical rainforest, Amazonia. Biological Conservation, 91:135-142.

Summers, P. M. 1998. Estoque, decomposição, e nutrientes da liteira grossa em floresta de terra-firme na Amazônia central. M.Sc. thesis, Instituto Nacional de Pesquisas da Amazônia (INPA), Manaus, Amazonas, Brasil. 94 pp.

Tabarelli, M.; Mantovani, W.; Peres, C. A. 1999. Effects of habitat fragmentation on plant guild structure in the montane Atlantic forest of southeastern Brazil. Biological Conservation, 91:119127.

Terborgh, J.; Lopes, L.; Tello, J.; Yu, D.; Bruni, A. R. 1997. Transitory states in relaxing ecosystems of land bridge islands. In: W. F. Laurance, R. O. Bierregaard (eds.). Tropical Forest Remnants: Ecology, Management, and Conservation of Fragmented Landscape. University of Chicago Press, Chicago, p. 256-274.
Tilman, D.; May, R. M.; Lehman, C. L.; Nowak, M. A. 1994. Habitat destruction and the extinction debt. Nature, 371:6566.

Turner, I. M.; Chua, K. S.; Ong, J.; Soong, B.; Tan H. 1996. A century of plant species loss from an isolated fragment of lowland tropical forest. Conservation Biology, 10:1229-1244.

Uhl, C.; Buschbacher, R.; Serrão, E. A. S. 1988. Abandoned pastures in eastern Amazonia. I. Patterns of plant succession. Journal of Ecology, 76:633-681.

Uhl, C.; Kauffman, J. B. 1990. Deforestation, fire susceptility, and potential tree responses to fire in the eastern Amazon. Ecology, 71:437-449.

Van Wagner, C. E. 1968. The line-intersect method in forest fuel sampling. Forest Science, 14:20-26.

Viana, V. M.; Tabanez, A. A. J.; Batista, J. L. F. 1997. Dynamics and restoration of forest fragments in the Brazilian Atlantic moist forest. In: W. F. Laurance, R. O. Bierregaard (eds.). Tropical Forest Remnants: Ecology, Management, and Conservation of Fragmented Landscape. University of Chicago Press, Chicago, p. 351-365.

Williams-Limera, G. 1990. Vegetative structure and environmental conditions of forest edges in Panama. Journal of Ecology, 78:356373.

Zudeima, P.A.; Sayer, J. A.; Dijkman, W. 1996. Forest fragmentation and biodiversity: the case for intermediate-sized conservation areas. Environmental Conservation, 23:290-297.

Recebido em 05/10/04

Aceito em 17/04/06 